\title{
IR OBSERVATIONS OF THE SYMBIOTIC NOVA HM SAGITTAE
}

\author{
O.G. Taranova, B.F. Yudin \\ Sternberg State Astronomical Institute \\ Moscow, USSR
}

The infrared photometry of the symbiotic nova HM Sge, with a Mira variable as a cool component, shows that since 1983 its mean IR brightness began to decrease from the previous post-outburst level (Figure 1). In 1988 the mean $J$ magnitude was already about 1 mag fainter than it had been until 1983.

An analysis of the photometric observations shows that the decrease in the mean IR light level of HM Sge may be understood in the framework of the commonly accepted model of this binary stellar system (Taranova and Yudin 1983), if we suggest that the Mira variable and the inner parts of its circumstellar envelope (RMira envelope $3 \times 10^{14} \mathrm{~cm}$; tans $\leqslant 1$ at $1.25 \mathrm{\mu m}$ until 1983) were gradually obscured by the dust originated between them and the observer.

The dust envelope surrounding the Mira variable in HM Sge is influenced by intense hard UV radiation from the hot component and by stellar wind. Consequently, it is possible that the Mira's envelope has not a spherical form. In this case the observed changes of the mean IR light level in HM Sge might be related to the orbital motion of this binary system. A more detailed discussion of this phenomenon, observed also in some other symbiotic Miras, was published by Whitelock (1987).

At the same time, IR emission from the dust envelope, swept op by the stellar wind of the erupted hot component of HM Sge and heated by its radiation ( $R_{\text {a wept }}$ up envelope $\geq 1013 \mathrm{~cm}, \tau_{\mathrm{l}} \leqslant 0.6$ ), did not change essentially during the last several years. At present the swept up dust envelope absorbs about $50 \%$ of the Lc-emission from the hot subdwarf, having a luminosity about equal to that of its cool

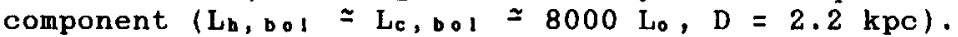




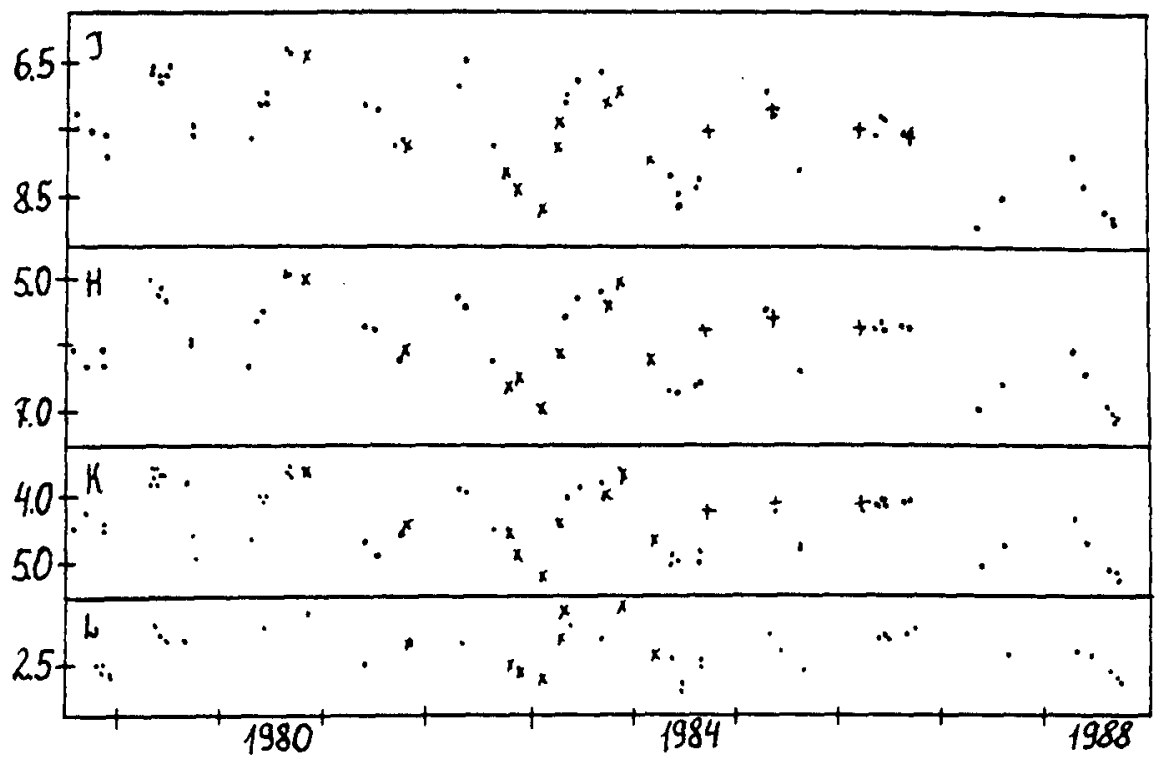

Fig. 1 JHKL light curves of FM Sge. Symbols are: (0) for our data; ( + ) date from Kenyon (1988); ( $x$ ) data from Lorenzetti et al. (1985).

\section{REFERENCES :}

Kenyon, S.J.: 1988, Astron. J. $\underline{96}, 337$.

Lorenzetti, D., Saraceno, P., Strafella, F.: 1985, Astrophys. J., 298, 350 .

Taranova, O.G., Yudin, B.F.: 1983, Astron. and Astrophys., 117, 209.

Whitelock, P.A.: 1987, Publ. Astron. Soc. Pac., 99, 573. 